Recepción: 22/11/2018

Aceptación: 19/12/2018

Publicación: 20/03/2019

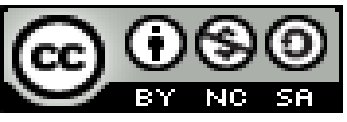

Ciencias de la educación

Artículo de investigación

\title{
Desde la educación humanista, hasta la escuela humanitaria
}

\section{From humanistic education, to humanitarian school}

\section{Da educação humanista à escola humanitária}

\author{
Oscar Darío Palacios-Acosta ${ }^{\mathrm{I}}$ \\ oscar.palacios@fundes.edu.co \\ Sandra María Saiz-Ucros II \\ sandra.saiz@fundes.edu.co
}

Correspondencia: oscar.palacios@fundes.edu.co

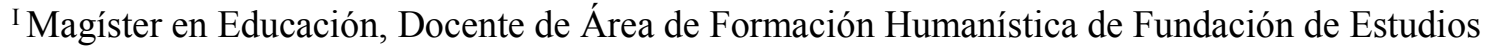
Superiores Monseñor Abraham Escudero Montoya. Tolima, Colombia.

${ }^{\text {II }}$ Máster Universitario en Liderazgo y Dirección de Centros Educativos, Director Programa Psicología de Fundación de Estudios Superiores Monseñor Abraham Escudero Montoya. Tolima, Colombia.
} 


\title{
Resumen
}

La pedagogía como fundamento del proyecto educativo, más que ofrecer herramientas para el desarrollo de procesos, tiene el compromiso de construir experiencias de vida. Así, se acoge la hipótesis de cómo un proyecto educativo basado en el desarrollo de competencias afectivas, puede intervenir no solo procesos cognitivos desde las habilidades para el aprendizaje, sino también vincular elementos pedagógicos para la reconstrucción de las estructuras sociales primarias; familia y escuela. Desde esta perspectiva, puede que haya un cambio de dirección frente a las cuatro preguntas básicas para la elaboración del currículo; ¿Qué enseñar?, ¿Cuándo enseñar?, ¿Cómo enseñar? y ¿Qué; cómo y cuándo evaluar? (Zubiría, 2014) para trascender al ¿Cómo desarrollar la actitud de querer aprender?, ¿Con qué actitud se debe enseñar y cómo se espera que respondan los estudiantes?, ¿Qué motivaciones tienen los estudiantes para querer aprender? y ¿Cómo evaluar su progreso en virtud de su formación como ser-humano? En resumen, desde un enfoque cualitativo-descriptivo-pre post facto, se pretende demostrar que al trabajar un proyecto educativo desde el sujeto como ser-afectivo, podría permitir a los directivos, docentes, padres de familia y estudiantes, asumir la educación como una experiencia de vida que permita revolucionar una educación humanista hacia la construcción de una escuela humanitaria.

Palabras clave: educación humanista; pedagogía; currículo; afectividad; escuela humanitaria.

\begin{abstract}
Pedagogy as the base of education, more than offering simple tools for the development of processes, engages the compromise of elaborating life experiences. Thus, this work assumes the hypothesis of how an educative project supported on the development of affective competences, may supply not only cognitive processes through learning abilities, but also to articulate pedagogical elements for the reconstruction of the primary social structures; family and school. In this way, it may be a change of the paradigm for curriculum designing: What to teach? When to teach? How to teach? And what and when to evaluate? (Zubiría, 2014), to transcend to How to develop the attitude of students to want to learn? What attitude must be taken to teach and how do teachers expect that students react? And how to evaluate their progress in virtue of his education such as a human-being?
\end{abstract}


In brief, from a qualitative-descriptive pre post facto approach, it will be demonstrated that working on an educative project concerning the subject as an affective-being, may help the principals, teachers, parents and students to assume education as a life experience which permits revolutionize humanist education towards the construction of a humanitarian school.

Keys words: humanist education; pedagogy; curriculum; affectivity; humanitarian school.

\section{Resumo}

A pedagogia como alicerce do projeto educacional, ao invés de oferecer ferramentas para o desenvolvimento de processos, está comprometida em construir experiências de vida. Assim, aceitamos a hipótese de como um projeto educativo, baseado no desenvolvimento de competências afetivas, pode não apenas intervir nos processos cognitivos a partir das habilidades de aprendizagem, mas também articular elementos pedagógicos para a reconstrução de estruturas sociais primárias; família e escola. Dessa perspectiva, pode haver uma mudança de direção diante das quatro questões básicas para a preparação do currículo; O que ensinar Quando ensinar Como ensinar? e que; como e quando avaliar? (Zubiría, 2014) para transcender o Como desenvolver a atitude de querer aprender?, Que atitude deve ser ensinada e como se espera que os alunos respondam? Que motivações os alunos precisam aprender? e Como avaliar seu progresso em virtude de seu treinamento como ser humano? Em suma, a partir de um post qualitativa abordagem facto pré-descritiva é mostrar que, trabalhando um projeto educacional do sujeito como ser-afetiva, poderia permitir que diretores, professores, pais e alunos assumir a educação como um experiência de vida que permite revolucionar uma educação humanista para a construção de uma escola humanitária.

Palavras-chave: educação humanista; pedagogia; currículo; afetividade; escola humanitária.

\section{Introducción}

La pedagogía, como fundamento teórico para la construcción del Proyecto Educativo (PE), debe ofrecer más que herramientas para el desarrollo de simples procesos, puesto que tiene el compromiso de construir experiencias de vida. En este documento, se acoge la hipótesis de cómo un PE basado en el desarrollo de competencias afectivas, puede favorecer no solo los procesos 
cognitivos desde el fortalecimiento de las habilidades para el aprendizaje, sino también vincular elementos pedagógicos para la reconstrucción de las estructuras sociales primarias como la familia y la escuela.

Partiendo de la concordancia entre diversos autores, frente al rol de los maestros como modeladores de cuestionamientos o preguntas, no se puede desconocer que el sentido de aprender está mucho más conexo con el hacer para encontrar las respuestas. Por ende; si bien el papel de la escuela es despertar la conciencia de juicio, la curiosidad, la creatividad, el raciocinio, el intelecto y el discernimiento, entre otros elementos dentro de su aparato académico, también tiene un Contrato Social (Riádigos, 2014; Hobbes, 1984) íntimamente comprometido con el desarrollo de habilidades como la empatía, la negociación, la gestión de conflictos y la observación crítica como herramientas fundamentales para la construcción de una sociedad local y global más justa y sostenible. De la misma forma, no se puede negar la responsabilidad que tiene la escuela, frente al desarrollo personal de los docentes y estudiantes desde sus Dimensiones Éticas (Kohlberg, 1989) entre ellas; su sentido de libertad, de afectividad, de intimidad, de protección a la naturaleza y de humanidad.

Desde esta perspectiva, puede que haya un cambio de dirección frente a las cuatro preguntas básicas para la elaboración del currículo; ¿Qué enseñar?, ¿Cuándo enseñar?, ¿Cómo enseñar? y ¿Qué; cómo y cuándo evaluar? (Zubiría, 2014 p.16) para trascender al ¿Cómo desarrollar la actitud de querer aprender?, ¿Con qué actitud se debe enseñar y cómo se espera que respondan los estudiantes?, ¿Qué motivaciones tienen los estudiantes para querer aprender? y ¿Cómo evaluar el progreso del estudiante en virtud de su formación como ser-humano?

\section{Introducción al problema}

Indistintamente a los niveles de formación ofrecidos por las Instituciones Educativas (IE), cada una de ellas tiene una Responsabilidad Social (RS) inherente a sus principios institucionales. Este tipo de compromisos, responden a la necesidad de establecer un puente racional entre el currículo y las necesidades reales del contexto, incluyendo tanto a las personas como al ecosistema, dentro del cual se construye el PE. De la misma forma, establecer un acuerdo de RS permite a las IE superar la condición egocéntrica de instituciones 'micro' para trascender en la forma de comunidades globales de aprendizaje ‘macro' (Vallaeys, 2009) y desde esta categoría, no solo 
comprometerse con el desarrollo intelectual de los estudiantes, sino con la gestión de un PE integral para la formación del ser-humano.

Así también, las IE tienen un Contrato Social (CS) que alega "el encuadre de las relaciones de convivencia entre los seres humanos de diferentes sociedades" (Riádigos, 2014. p. 481) sin el cual, las IE estarían sumidas en un estado de convivencia ficticia, acuñada a políticas forzadas mediante la naturalización de la desigualdad y la imposición de dogmas. No hay que olvidar, que uno de los principios fundamentales de cualquier PE es ofrecer una educación orientada en favor de la justicia y la participación democrática, mediante procesos funcionales que favorezcan el desarrollo de experiencias de aprendizaje significativas, coherentes con las necesidades actuales. (Riádigos, 2014)

Desde esta perspectiva, para pensadores como Hume, Smith, Bentham y Mill (Riádigos, 2014. p. 486) el principio de 'justicia' es admisible solo cuando las estructuras sociales se ordenan debidamente, dando un consentimiento tácito a sus sistemas de gobierno, dimitiendo algunas libertades para cohabitar en armonía y donde el bienestar social dependa de la suma del bienestar de todos sus integrantes. Por lo tanto, un PE justo, debe construir su CS en el sentido de propender por el bienestar de toda la comunidad educativa.

Es por esto que la Fundación de Estudios Superiores Monseñor Abraham Escudero Montoya FUNDES, en un trabajo conjunto de casi tres años con la Institución Educativa San Isidoro del Municipio del Espinal (Tolima) y de un año con la Institución Educativa Manuel Elkin Patarroyo del Municipio de Girardot (Cundinamarca), se preocupó por tipificar factores condicionantes del estado personal y emocional, con poblaciones muestrales de estudiantes de básica secundaria y media, relacionados con el ambiente de enseñanza-aprendizaje, el entorno familiar, el entorno educativo y el ambiente escolar, para determinar el estado de relación existente entre el currículo y el contexto de los educandos.

Frente a los resultados obtenidos, en la institución tolimense se encontró que, el 59\% de los participantes asume que la inestabilidad laboral de sus padres afecta directamente la permanencia de los estudiantes en el aula y por ende la continuidad de sus procesos académicos. Asimismo, dentro del entorno familiar, el $65 \%$ reconoce que las principales causas de su bajo rendimiento 
académico, resulta de la necesidad de trabajar para aportar en sus hogares, los continuos casos de maternidad/paternidad temprana (sobre todo en el nivel de educación media) y el consumo o presencia de consumidores de sustancias psicoactivas legales y/o ilegales en sus hogares. De la misma forma, en cuanto al entorno educativo, el 59\% advierte poco gusto por el estudio, escasa motivación frente al desarrollo de los planes de asignatura y relaciones interpersonales poco afectivas entre docentes-estudiantes y estudiantes-estudiantes. Finalmente, dentro del ambiente escolar, el $48 \%$ de los participantes han tenido llamados de atención por problemas de convivencia, conflictos entre compañeros o la reprobación reiterada del año escolar.

Por su parte, la institución Manuel Elkin Patarroyo, para el año 2017 aplicó un instrumento de autoevaluación tipo encuesta denominado: Identificación de las causas por las cuales los estudiantes tienen bajo rendimiento académico diseñado por el departamento de Psicología de la misma institución; mediante el cual se logró obtener los siguientes resultados; un 65\% los estudiantes afirmaron tener problemas para entender las instrucciones de los docentes, un $45 \%$ manifestó que no le gusta la metodología utilizada por los docentes, un 25\% manifestó que no hay buenas relaciones interpersonales entre docentes y estudiantes, un $47 \%$ afirmó que el sistema de evaluación es complejo y poco claro. Adicionalmente, estos resultaron demostraron que el $75 \%$ de docentes aplican una metodología tradicional de corte magistral, desconociendo el enfoque institucional definido por el PEI, sólo el $24 \%$ aplica el modelo educativo institucional y el $1 \%$ utiliza un modelo ecléctico desde la implementación de una variedad de ideas en su quehacer pedagógico.

Realizado el diagnóstico en ambas instituciones, se retomaron los resultados para cotejar similitudes, focalizando patrones categóricos de tipo directivo-institucional, de disparidad metodológica y de cooperación/interacción entre docentes-estudiantes, estudiantes-estudiantes y familia-escuela. De esta manera, el tipo de reflexión que se hace necesaria, conlleva a examinar los modelos pedagógicos institucionales para acordar desde la dirección, un trabajo de retroalimentación de los resultados obtenidos, que permita considerar y definir políticas educativas para generar acciones contextuales, que procuren la permanencia de los estudiantes, logren disminuir la deserción, aseguren la continuidad educativa y por consiguiente propendan una mejora sobre los índices de calidad educativa. 
No obstante, si bien las condiciones de las instituciones educativas mencionadas anteriormente, no dejan de ser inquietantes, desafortunadamente no son ajenas al contexto nacional. En estudios previos, como el publicado por la Friends United Foundation y su Departamento de Analistas en Violencia Juvenil y Delitos Contra Menores de Edad, para la edición del 12 de noviembre del 2013 del Diario El Espectador, se percibe una fuerte preocupación por el aumento en los índices de violencia escolar, de acuerdo con el número de casos presentados en las principales ciudades de Colombia. En dicho informe, se afirma que los casos más frecuentes se presentan por homofobia $30 \%$, discriminación racial 25\%, "barrismo" o fanatismo deportivo principalmente relacionado con equipos de fútbol $20 \%$, matoneo por algún tipo de discapacidad $10 \%$, matoneo por apariencia física 10\% y otros tipos de matoneo fuera de las razones antes presentadas 5\%. Así mismo, en otro artículo publicado en el Diario el País en su edición del 23 de Junio de 2014, la Fundación PLAN de Colombia afirmó que luego de realizar un trabajo con 28.967 estudiantes de instituciones educativas públicas de los niveles de primaria y bachillerato, en diferentes municipios del Cauca, Valle del Cauca, Nariño, Chocó, Sucre y Bolívar se encontró que el $75,5 \%$ de los estudiantes habían sido víctima de algún tipo de violencia escolar, principalmente “bullying”, entre estos el 78\% niños y el 77\% niñas.

Posteriormente, en un contexto mucho más amplio, en la edición del 17 de enero de 2017 del Diario El Tiempo, se publicó un informe de la UNESCO titulado "Dos de cada 10 alumnos en el mundo sufren acoso y violencia escolar", donde se afirma que 246 millones de niños y jóvenes de todo el mundo, han sido víctimas de algún tipo de violencia escolar. Igualmente, el 34\% de niños y niñas entre los 11 y 13 años argumentaron haber sido acosados durante periodos de tiempo prolongados previos al estudio, mientras que un $8 \%$ de la población encuestada afirmó estar bajo acoso diario. Bajo estos términos, la Directora General de la Organización de la ONU para la Educación, la Ciencia y la Cultural - UNESCO, se pronunció manifestando que lo anterior determina un estado de violación grave del Derecho a la Educación y que los centros de aprendizaje deben convertirse en espacios seguros para todos.

Por otro lado, desde la Universidad San Buenaventura, Extensión Armenia, en el año 2015 se mostró un informe longitudinal, sobre el índice de consumo de Sustancias Psicoactivas (SPA) en escolares del Departamento del Quindío entre los años 2009 a 2012. En este trabajo, se 
argumenta que de acuerdo con el II Estudio de consumo de sustancias psicoactivas en estudiantes de secundaria presentado por el Ministerio de Justicia y del Derecho, el Ministerio de Salud y Protección Social y el Ministerio de Educación Nacional, para el 2012, el 40\% de los estudiantes encuestados respondieron afirmativamente frente al consumo de bebidas alcohólicas; el 24,3\% aceptó haber consumido tabaco alguna vez en su vida y el 7\% afirmó haber consumido mariguana para efectos recreativos (Andrade y Torres, 2015).

Para el Departamento del Quindío, se descubrió que la edad promedio para iniciar el consumo de SPA son los 12 años, con una frecuencia de consumo de más de tres veces al día en un $25 \%$ y otro $25 \%$ con una frecuencia de consumo de una o dos veces al día (Gonzáles, Giraldo, et al., 2011). Con respecto a las conductas de riesgo que presentan los adolescentes consumidores, se encontraron principalmente los embarazos prematuros, la explotación sexual, contagio de enfermedades por transmisión sexual, el abandono escolar y la comisión de delitos (Andrade et al. 2010). Así mismo, se identificaron factores comunes entre los jóvenes consumidores, tales como la falta de autoestima, la presión de pares, crisis emocionales, familias disfuncionales y el estado de convivencia con otros consumidores (Andrade y Torres, 2015).

De igual manera, otro estudio publicado desde la Universidad Mariana de Pasto para el año 2016, relacionó un aumento en el índice de consumo de SPA en estudiantes de educación media a partir del año 2013 , con un incremento del $1,5 \%$ al $4,4 \%$ en el consumo de mariguana y del $0,4 \%$ al $1,2 \%$ para el caso de las drogas sintéticas. Dentro de las conclusiones de este estudio, se puede observar que la edad promedio de consumo con relación a los estudiantes de décimo y undécimo grado de secundaria, son los 15,5 años de edad con agravantes vinculados a comportamientos disociativos a nivel interpersonal sobre todo en el hogar y la escuela (Santacruz et al., 2015). Sin embargo, se vislumbra la posibilidad de encontrar refuerzos positivos con relación a los buenos lazos sociales entre familiares, amigos y compañeros de escuela, la posibilidad de establecer normas de comportamiento claras y el fortalecimiento de las habilidades sociales como la empatía, la resiliencia y la capacidad de solucionar conflictos (Santacruz, 2015).

Adicionalmente, en otro estudio sobre los factores psicosociales relacionados con el consumo de alcohol en adolescentes escolarizados, presentado desde la Pontificia Universidad Javeriana seccional Cali (Valle del Cauca) para el primer semestre del 2018, se observa que el 67,5\% de 
una muestra de estudiantes encuestados por el Observatorio de Drogas de Colombia ha consumido alcohol en el último año. De acuerdo con este estudio, tanto el grado como la frecuencia de consumo aumentaron en proporción directa a la edad de los escolares y del grado de escolaridad. Tanto así, que entre los jóvenes de 11 y 12 años se reportó un 20\% de consumidores, mientras que entre los adolescentes de 16 a 18 años se reportó un 58,1\%. Además, el 35\% de la población consumidora reportó un nivel de consumo de alto riesgo, de acuerdo con los estándares del Ministerio de Salud y Protección Social de Colombia para 2017 (Orcasita et al., 2018). Entre los factores de riesgo asociados a los altos niveles de consumo de alcohol entre los jóvenes, fueron identificados la influencia de las familias como pares de consumo, la aceptación y permisividad social de consumo en edades tempranas, estructuras familiares disfuncionales, la tolerancia y naturalización del consumo de alcohol por parte de los padres y la presión grupal entre pares (Orcasita et al., 2018).

No obstante, también se mencionan algunas alternativas de protección frente al consumo de bebidas alcohólicas; entre ellas, una buena relación afectiva y lazos de comunicación estrechos entre padres e hijos, la adaptabilidad familiar para flexibilizar las normas de convivencia, el fortalecimiento del vínculo afectivo entre padres e hijos y la cohesión familiar. A nivel escolar, se pueden favorecer los programas de apoyo frente a los estilos de crianza, la coexistencia de normas y conductas claras tanto a nivel de familia como de escuela (Orcasita et al., 2018).

Desde otra perspectiva, recabando cifras sobre los casos de violencia intrafamiliar con incidencia en población infantil reportados en los últimos dos años, los porcentajes tampoco se muestran muy halagadores. Para la edición del 2 de septiembre de 2016, la revista Semana, sección Nación, se muestra un artículo titulado "La epidemia de la violencia intrafamiliar". En este, se recogen los datos sobre los diferentes casos reportados al Instituto Nacional de Medicina Legal y Ciencias Forenses de Colombia hasta el 31 de julio del mismo año, llegando a 44,796 casos de los cuales los departamentos con mayor índice de reportes fueron; Antioquia (4.576), Cundinamarca (3.471), Valle del Cauca (3.029), Santander (2.413), Atlántico (2.178), Boyacá (1.613), Meta (1.577), Norte de Santander (1.262), Bolívar (1.232) y Tolima (1.204) y una cifra exorbitante en la capital del país con 11,687 casos. Dentro del mismo reporte, se muestra que el 45\% de las veces fueron denunciados como violencia ocasional, el $21 \%$ de periodicidad frecuente y el 15\% 
de violencia constante, aclarando que, el $25 \%$ de las víctimas fueron mujeres y el $8 \%$ fueron menores de edad.

Dichos datos, fueron corroborados por el Informe Forensis 2016 del Instituto Nacional de Medicina Legal de Colombia, donde también se muestra que, para ese mismo año, se identificaron 26,473 casos de violencia intrafamiliar sin incluir la violencia de pareja, dentro de los cuales el $38.08 \%$ de las víctimas fueron menores de edad. Así mismo, se afirma que los casos más frecuentes de violencia se cometieron contra menores entre los 10 y 14 años en un 33\% y donde se asume como grupo de mayor riesgo a los adolescentes entre los 15 y 17 años con un 97.99\% en una taza por cien mil habitantes (Montoya, 2016. pp. 211). De esta manera, los departamentos con mayor índice de violencia intrafamiliar según el número de casos denunciados para 2016 fueron; Antioquia (936), Cundinamarca (930), Meta (480), Santander (475), Valle del Cauca (460) y Boyacá (367). Así mismo, las capitales con mayor índice de casos denunciados fueron Bogotá D. C. (3,473), Medellín (519) y Villavicencio (385). (Montoya, 2016. pp. 216240)

Finalmente, el diario El Espectador, en su edición del 8 de marzo de 2018, sección Nacional, presentó el artículo "Cada 28 minutos una mujer es víctima de violencia de género en Colombia". En esa edición fue posible observar que, aunque se presentaron 441 casos menos que en el primer semestre de 2017, las cifras no dejaron de ser preocupantes. Para este reporte se presentaron 1,716 casos de violencia doméstica, principalmente ejercida por parte de los exnovios o exesposos principalmente dentro del hogar, aunque hubo reportes de casos que se presentaron en la vía pública. En cuanto a los Departamentos con mayor porcentaje de reportes reinciden Antioquia, Cundinamarca, Valle del Cauca, Santander y Meta, uniéndose a la lista el departamento del Atlántico.

Por consiguiente, se valida como apremiante, el diseño de un currículo que permita fortalecer las competencias afectivas y las habilidades sociales de las nuevas generaciones, no solo desde el componente teórico-intelectual-educativo, sino también desde la virtud de la ética y la sensibilidad del saber-ser. Consecuentemente, la propuesta educativa derivada de esta investigación se articula a partir de tres elementos fundamentales; lo cognitivo, lo social y lo afectivo; sugiriendo atender un modelo pedagógico de formación integral, desde la perspectiva 
sujeto-familia-escuela, asumiendo el siguiente reto: ¿Cómo desarrollar las competencias afectivas como alternativa pedagógica para la reconstrucción de las estructuras sociales básicas (familia y escuela) desde la concepción de una escuela humanitaria?

\section{Importancia del Problema}

La preocupación por integrar un componente socio-emocional en los procesos de enseñanzaaprendizaje no es algo literalmente nuevo, de hecho; se han propiciado diversos espacios de reflexión tanto a nivel nacional como internacional sobre cómo, cuándo y por qué es necesario integralizar los componentes socio-cognitivos y socio-afectivos dentro de un mismo plan de acción pedagógica. Haciendo una muy breve reseña sobre los factores influyentes en el diseño y administración del quehacer educativo en las aulas, es posible identificar una fuerte influencia política, frente a la necesidad de competir en el mercado laboral y frente a la gestión de proyectos estatales para el desarrollo económico, tecnológico y científico.

En principio; porque durante las décadas del cincuenta y mediados del sesenta, el boom de la exploración espacial abrió campo al desarrollo de métodos y estrategias para el óptimo aprendizaje de las matemáticas y las ciencias (Olson y Wyett, 2000). Por ende, la estructura académica de los proyectos educativos, fue diseñada primordialmente, para el desarrollo de programas científicos orientados hacia el campo de la física, la ingeniería y la aerodinámica. Posteriormente, entre las décadas del sesenta y setenta, se inclinaron hacia el rescate de la figura afectiva del maestro, pero descuidando el componente cognitivo; lo cual no permitió que se obtuvieran los resultados esperados (García, 2009).

Pero, fue a partir de la década del ochenta que se percibe, de forma casi obligada, la necesidad de integralizar un componente socio-afectivo en el diseño, elaboración e implementación del PE. De esta manera, es como se ha venido hablando de estrategias cognitivas, metacognitivas, autoregulatorias, para la solución de conflictos, entre otras (García, 2009). De forma semejante, durante los últimos diez años, se ha incrementado el interés de los maestros por incorporar otro tipo de competencias paralelas al campo cognitivo, frente a la necesidad de fortalecer la dimensión afectiva de los docentes bajo dos condiciones esenciales; la empatía y la forma de proyectar dicha empatía a los estudiantes (García, 2009). Aunando este concepto a estudios 
previos, bien podría afirmarse que este tipo de empatía corresponde a lo que Gardner (1998) reconoce como "Inteligencia intra e interpersonal", la misma que posteriormente Goleman(1995) identificaría como "Inteligencia Emocional", actualmente considerada como la herramienta esencial para generar climas sociales propicios para el aprendizaje (García, 2009).

Igualmente, las estructuras educativas estatales siempre han considerado los factores de promoción para el campo laboral. Así, la preocupación por desarrollar un PE que no solo converja en el saber-aprender y en el saber-hacer, subyace de la necesidad de formar una planta laboral que sepa saber-ser y saber-convivir en grupo, tal como se observa en el Manual para el Desarrollo de Habilidades Socioemocionales en Planteles de Educación Media Superior de la Secretaria de Educación Pública del Estado de México (SEP, 2014). Desde este plano de observación, se reconoce que los empresarios mexicanos, tienen una alta apreciación hacia los empleados que demuestran buenas habilidades sociales en lo concerniente a mantener una buena capacidad de comunicación, de innovación y de eficiencia personal. (pp. 19-20)

Adicionalmente, dentro de las bases del Plan Nacional de Desarrollo 2014 - 2018 para la Republica de Colombia (PND), propuesto por el Presidente Juan Manuel Santos, bajo la dirección de Simón Gaviria Muñoz, Director General del Departamento Nacional de Planeación (DNP) se reconoce que, si bien la educación es el instrumento más poderoso para cerrar las actuales brechas sociales; lograr avances en términos de movilidad socio-laboral, mejorar la vida de los colombianos con menores ingresos y la baja calidad de todos los niveles educativos, sigue siendo una problemática que limita la formación y el desarrollo de competencias para el trabajo y para la vida. (p.67)

También, reconoce que los retos educativos de la coyuntura mundial actual, convergen en la actual demanda de un capital humano bien informado, innovador, crítico, flexible, plurilingüe, que aporte en los procesos de transformación económica y social, con una sólida conciencia ambiental que le permita interactuar con su entorno, capaz de manejar el riesgo y que aporte a los procesos de desarrollo humano sostenible. Pero también, debe ser capaz de interactuar con otros, manejar conflictos de manera constructiva y respetar los procesos de participación democrática (p.72). 
Igualmente, en el documento matriz del Plan Nacional Decenal de Educación 2016 - 2026 (PNDE), propuesto por el Ministerio de Educación Nacional de Colombia (MEN) se reitera que después de cincuenta años de conflicto, la educación es el camino para consolidar una verdadera paz; lo cual exige la formación de buenos ciudadanos, capaces de resolver conflictos de forma pacífica, reflexivos y abiertos al diálogo, capaces de estimular espacios propicios para mantener una sana convivencia (p.9).

De ahí que la visión del PNDE colombiano para el 2026, insista en que uno de los principales retos del MEN es impulsar un PE que logre transformar el paradigma tradicional que ha dominado la educación hasta el momento; que transforme la condición de una pedagogía basada en la transmisión de saberes y la convierta en una propuesta de educación integral, orientada hacia el desarrollo humano y la construcción de una nación en términos de diversidad cultural, social y de creciente internacionalización (p.18). Por lo tanto, es necesario promover la creatividad individual y colectiva, el deseo y la voluntad de aprender, el pensamiento crítico y el desarrollo de las competencias emocionales, además de una conciencia ética que permita orientar acciones sobre la base de la solidaridad, el respeto mutuo, el trabajo autónomo y responsable, el reconocimiento y cuidado de la naturaleza, y la riqueza territorial, étnica y cultural de la nación. (ibíd.)

Adicionalmente, el PNDE asume el reto de transformar la concepción de currículo como uno de sus alcances a 2026. Esta propuesta, se ajusta a una construcción de lineamientos curriculares comprometidos con el reconocimiento de la diversidad de los contextos sociales y con la formación de ciudadanos críticos, creativos, con sólidos valores éticos, respetuosos de la diversidad y dispuestos a participar de forma democrática y productiva en la vida social. Asimismo; fundamentados en el respeto a las diferentes culturas regionales y locales, capaces de reconocer los procesos de mundialización de la economía, la cultura, la política, la ciencia y la tecnología. (p.19)

Con todo lo anterior, el MEN persigue una articulación que implica el impulso de las competencias básicas; comunicativas y socioemocionales, para garantizar el desarrollo de competencias para la vida. Esto incluye las dimensiones sociales, subjetivas, cognitivas, técnicas, del lenguaje y la comunicación, del respeto por el otro, de la sensibilidad social y estética y del 
reconocimiento y disposición de convivir con aquellos que tienen diferencias culturales, religiosas, políticas, de género, entre otras, mientras no implique renunciar a los principios de equidad o a la negación de los derechos ciudadanos. (p.19)

Por consiguiente, la validez de este estudio se fundamenta tanto en las necesidades políticas de un Estado que propende por el mejoramiento de su sistema educativo, así como en las necesidades educativas que asume la escuela. El desarrollo de una propuesta educativa con fundamento socioafectivo-cognitivo, corresponde indistintamente a los planes de mejora educativa desde lo social, político, económico, científico, tecnológico o cultural.

\section{Metodología}

Esta propuesta, fue diseñada desde un enfoque cualitativo de tipo descriptivo pre post facto (Kothary \& Garg, 2014), considerando que los autores no tienen control sobre las variables y solo se pueden evaluar desde lo que ha sucedido, frente a lo que está sucediendo. Desde esta perspectiva, el trabajo se fundamenta en la observación de dos modelos de comportamiento socio-académico en instituciones educativas públicas de carácter urbano; uno focalizado hacia los estudiantes y el otro hacia el componente directivo-administrativo. Cabe resaltar que, para establecer el modelo diagnóstico de las dos instituciones educativas, se analizaron algunos datos estadísticos de tipo cuantitativo, tanto para identificar porcentajes de deserción escolar y reprobación académica, como para identificar y tipificar factores condicionantes del estado personal y socioafectivo de los estudiantes.

De esta manera, se implementó un trabajo diagnóstico en a la Institución Educativa San Isidoro del Municipio del Espinal, departamento del Tolima; el cual se compone de cuatro sedes con niveles de educación preescolar y básica primaria y una sede central de educación básica secundaria y media. La institución cuenta con tres jornadas de atención a estudiantes; mañana, tarde y fin de semana; incluyendo una población total de 3236 educandos, 99 docentes, 6 coordinadores, 2 docentes de apoyo y 1 rector. Durante las vigencias 2016 y 2017, la institución había realizado dos ejercicios diagnósticos, en los que se aplicaron dos instrumentos diseñados bajo el modelo de encuesta estructurada utilizando la escala de Likert, descritos de la siguiente manera: 
Para el segundo semestre de la vigencia 2016, se aplicó la 'Encuesta diagnóstica de deserción escolar' compuesta por 8 ítems de información personal y 20 ítems diseñados para identificar factores asociados a la deserción escolar discriminados en cuatro categorías; factores ambientales, entorno familiar, ambiente escolar y entorno educativo. Siguiendo el mismo procedimiento, para el primer semestre de la vigencia 2017, se aplicó el segundo instrumento, denominado 'Encuesta para identificar factores determinantes sobre los procesos de enseñanzaaprendizaje', con la diferencia de que este último, fue únicamente dirigido a población estudiantil en el nivel de educación media (grados décimo y undécimo); considerando que los estudiantes de este nivel, son la representación del proceso académico, resultante del modelo pedagógico institucional. Este segundo instrumento, estuvo compuesto por 7 ítems de información personal, 19 ítems sobre el aprovechamiento del tiempo libre, 14 ítems de análisis reflexivo sobre los procesos de enseñanza aprendizaje y 11 ítems para identificar factores que inciden en la condición personal y emocional de los estudiantes. (Fuente propia, 2016; 2017)

Por otra parte, se realizó una segunda observación en la Institución Educativa Manuel Elkin Patarroyo del Municipio de Girardot, departamento de Cundinamarca; la cual cuenta con una sede de educación básica primaria que ofrece dos jornadas (mañana y tarde), una sede de educación básica primaria que ofrece una sola jornada (mañana) y una sede central con una sola jornada (mañana), para una población aproximada de 2000 estudiantes, 58 docentes, 3 coordinadores, 1 psicóloga y 1 rector.

Teniendo en cuenta lo anterior y apoyados en el marco directivo de la institución, se discriminaron dos tipos de análisis. En principio, se diagnosticaron el nivel profesional de los docentes vinculados, la condición socioeconómica de los padres de familia y los tipos de composición de las familias de los estudiantes. Para esta parte, se utilizaron las bases de datos del SIMAT (Sistema Integrado de Matricula) y las bases de datos institucionales. Por otra parte, se implementó un diagnostico frente a los índices de reprobación escolar y sobre las causas de bajo rendimiento académico por parte de los estudiantes. Para esto, se implementó un instrumento tipo encuesta, diseñado por la oficina de psicología de la institución, denominado 'Identificación de las causas por las cuales los estudiantes tienen bajo rendimiento académico', dentro del cual se 
categorizan los siguientes componentes: razones de tipo personal, razones de tipo institucional y razones de tipo familiar y/o de compañeros de curso.

\section{Resultados}

Para el año 2016, la Institución Educativa San Isidoro, mediante análisis cuantitativo, logró identificar los factores con mayor incidencia frente a los porcentajes de deserción escolar, categorizándolos en cuatro ambientes, de la siguiente manera: Factores ambientales; cambio de residencia 24,42\%, vivir lejos del colegio 23,55\%. Entorno Familiar; necesidad de trabajar para ayudar en el hogar $38,95 \%$, situación de maternidad/paternidad temprana $28,49 \%$, consumo frecuente de sustancias psicoactivas $25,87 \%$. Ambiente escolar; problemas de comportamiento o disciplinarios 40,7\%, dificultades académicas 28,49\%, conflictos entre compañeros 25,29\%, reprobación continúa del año escolar 25,29\%. Entorno educativo; poco gusto por el estudio $47,96 \%$.

De lo cual, haciendo un análisis paralelo sobre los hallazgos encontrados en este primer ejercicio, frente a los datos proporcionados por el Ministerio de Educación Nacional de Colombia (MEN) en el informe del Índice Sintético de Calidad Educativa (ISCE) de 2017, se aprecia que para la vigencia 2016, la institución educativa había logrado superar la Meta de Mejoramiento Anual (MMA) por 0.9 puntos, mientras que para la vigencia 2017, se ubicó 1.51 puntos por debajo de la misma. Es decir, se observa una posible relación entre el porcentaje de estudiantes que en 2016, afirmaron tener poco gusto por el estudio y los cambios en proporción negativa, frente a los indicadores de calidad educativa obtenidos para 2017. Además, esta misma relación, podría estar influenciando los comportamientos disociativos identificados dentro de la institución educativa, así como también podría estar promoviendo los demás comportamientos de riesgo mencionados en la tabla; con excepción de los descritos como 'factores ambientales', por corresponder mayormente al comportamiento de los índices nacionales de migración familiar por factores sociales y/o laborales.

Por otra parte, los datos obtenidos en 2017, sobre el segundo ejercicio orientado a identificar factores determinantes sobre los procesos de enseñanza-aprendizaje, aportaron los siguientes resultados. En cuanto a la población de estudio referenciada; el 50,4\% corresponde al género masculino, el 47, 2\% al femenino y el 3,3\% a la población LGBTI. Así también; el 70\% se 
encuentra en un rango de edad entre los 14 y 17 años y el 30\% entre los 18 y 20 años de edad. En cuanto a la estratificación socioeconómica, el 20,3\% corresponde al estrato1; el 52,8\% al estrato 2 ; el $14,6 \%$ al estrato 3 y el 12,2\% al estrato 4. Por ende, el rango poblacional de la institución se inclina hacia el nivel socioeconómico bajo. Dentro de la misma población, el 37,4\% asegura dedicarle la mayor parte del tiempo al estudio; el 50,4\% estudia y trabaja; el 4,9\% dedica mayor tiempo al trabajo; el 2,4\% se dedica mayormente a las labores del hogar y el último 4,9\% asevera dedicar la mayor parte de su tiempo al ocio.

Posteriormente, dentro del estudio correspondiente al uso del tiempo libre, se puede observar que entre las actividades más frecuentes entre los jóvenes encuestados, se encuentran: escuchar música (play lists) 79.7\%; chatear 47,2\%; ver televisión 44,7\%; hacer deporte $44,7 \%$ y salir con amigos 39,8\%. En sentido opuesto a las anteriores, las actividades reconocidas como poco o nada frecuentes fueron: visitar museos o lugares históricos $82 \%$; ir a cine o asistir a conciertos 57,7\%; jugar videojuegos o juegos de rol 52,8\%; escuchar la radio (programas no musicales o de opinión) 50,4\% y descansar 42,3\%. Adicionalmente, dentro del análisis sobre los procesos de enseñanza aprendizaje, fue posible categorizar los ítems de apreciación como favorables, intermedios o poco favorables (ver anexos; Tabla 1). Finalmente, el análisis de los datos concernientes a la categoría 'factores que inciden en la condición personal y emocional de los estudiantes', identifican como factores de alta incidencia a los padres de familia 79,7\%; hermanos mayores $62,6 \%$, las relaciones interpersonales fuera del colegio 55,3\%; la condición física del colegio $50,4 \%$ y a los docentes con un $44,7 \%$.

Por otro lado, en la Institución Educativa Manuel Elkin Patarroyo, dentro del primer ejercicio diagnóstico llevado a cabo en el año 2017, se encontró que el personal docente tiene características heterogéneas en un alto porcentaje, demostrando que el $80 \%$ de los maestros son licenciados en áreas específicas, dentro de los cuales el 45\% son especialista, el 20\% presenta un título de maestría y solo el 1\% del personal vinculado a la institución ostenta un título de doctorado. Por su parte; con respecto a la condición socioeconómica de los padres de familia, se observa que un $20 \%$ son empleados del sector comercial y hotelero, y un $60 \%$ cuenta con negocios independientes o trabajan en oficios varios. No obstante, también se percibe un $20 \%$ entre población desempleada o pensionada. En cuanto a la formación académica de los padres de 
familia, el $55 \%$ no termino los estudios primarios; otro $10 \%$ no terminaron los estudios secundarios; el 10\% de los padres de familia o acudientes han cursado algún tipo de estudios a nivel técnico; el 5\% a cursado algún tipo de estudios a nivel de educación superior y el 8\% han atendido cursos de educación informal de diversa índole, para mejorar los ingresos económicos familiares. Finalmente, en cuanto al perfil familiar de los estudiantes, se encontró que un 70\% corresponde a familias de padres separados, con solo un $30 \%$ de estudiantes provenientes de hogares nucleares; de éstos el 30\% son familias recontextualizadas y el 40\% componen hogares uniparentales.

Con relación al contexto académico de los educandos, para el año 2015 reprobaron 150 estudiantes y más de 200 quedaron pendientes por realizar actividades de recuperación en diferentes áreas. Para el 2016, reprobaron 220 estudiantes, quedando pendientes 330 para actividades de recuperación en diferentes áreas. Mientras que para el año 2017, la reprobación fue de 280 estudiantes, con más de 350 estudiantes por definir su situación mediante actividades de recuperación en diferentes áreas. Es decir, con relación a la vigencia 2015, el índice de reprobación ha aumentado en un 46,4\% y en un 42,9\% aproximadamente, para los estudiantes que definen su situación mediante procesos de recuperación en diferentes áreas.

Cabe señalar, que posterior a la aplicación del instrumento 'encuesta para la identificación de las causas por las cuales los estudiantes tienen bajo rendimiento académico’ implementado para la vigencia 2017, se encontraron los siguientes resultados: un 65\% de los estudiantes manifiesta que no comprenden las instrucciones de los docentes, un 45\% asevera que no le gusta la metodología utilizada por los maestros, un $25 \%$ advierten que no existen buenas relaciones interpersonales entre docentes y estudiantes y un $47 \%$ afirma que no hay claridad frente al sistema de evaluación. Así mismo, los resultados de demuestran que el 75\% de los maestros aplican una metodología tradicional, desconociendo el enfoque institucional; el 24\% de los docentes aplica el modelo pedagógico institucional y el $1 \%$ utiliza un modelo ecléctico, fundamentado en la implementación de varias ideas para su quehacer pedagógico.

Terminado el diagnóstico, se clasificaron los datos obtenidos, dentro de los cuales y de acuerdo con los intereses de la observación propuesta para esta institución, se dio prevalencia a la categoría 'razones institucionales'. En ésta, los estudiantes identificaron fallas en la metodología 
utilizada para el desarrollo de las clases, así como relaciones precarias entre docentes y estudiantes. Este tipo de resultados, han permitido generar desde la dirección, implementar estrategias para revisar la pertinencia del modelo pedagógico y replantear algunas políticas educativas dentro del Proyecto Educativo Institucional. Es decir, demostrar desde las directivas, que pese a los resultados encontrados, persiste un gran interés por determinar acciones en contexto, que procuren la permanencia de los estudiantes, disminuir la deserción escolar, asegurar la continuidad educativa y por consiguiente mejorar el ISCE, todo con el único propósito de corresponder a las necesidades reales de la comunidad educativa. Desde esta perspectiva, tanto el análisis sobre los procesos de enseñanza aprendizaje, como de los factores incidentes en la condición socioemocional del estudiantado, permiten validar la importancia del enfoque pedagógico y curricular frente a su relación con el desarrollo socioafectivo y psicoactivo del estudiantado. De la misma forma, se está validando la relación sobre la cual se construye esta propuesta; familia-sujeto-escuela.

\section{Discusión}

Para entrar en materia de discusión, es necesario concernir tres puntos de referencia que sustentan la hipótesis de esta investigación. En primer lugar, es evidente que el resultado del proceso pedagógico institucional, se relaciona congruentemente $\mathrm{y}$ directamente, con el estado motivacional de los educandos. Ya se ha dicho, que la escuela no puede quedar relegada únicamente al desarrollo intelectual de los estudiantes, y que éstos, además de inteligentes son emocionales. También se ha dicho, que la escuela debe asumir la responsabilidad de superar el concepto de estandarización pedagógica, también así, para los componentes estratégicos y didácticos de los procesos de enseñanza. De esta forma, las instituciones educativas, desde sus políticas, planes estratégicos y diseños metodológicos, tienen el reto de corresponder al principio de 'particularidad' que determina el sentido de ser-humano y de ser-inteligente [considerando que hacer lo contrario, podría tener una repercusión nociva para el desarrollo afectivo-cognitivo de los niños y jóvenes de diariamente habitan las aulas]. (Samper, 2009)

En segundo lugar, de acuerdo con los datos analizados anteriormente, queda establecido que los docentes y padres de familia son elementos altamente influyentes de la condición emocional de los estudiantes. Así, se confirma que la razón y el sentido misional de la 'escuela' trasciende las 
aulas, articulando el quehacer educativo dentro de un entorno de participación afectivopedagógico, compuesto de múltiples variables que cooperan unas con otras, dentro de las cuales, ha sido posible identificar hasta ahora; la relación entre padres-estudiantes; docentes-estudiantes; entorno educativo-estudiantes; currículo-estudiantes y estudiantes-estudiantes. Vale recordar las palabras de Maturana (2002) cuando explicita que 'el amor' como emoción, constituye el dominio de los parámetros de aceptación recíproca entre unos y otros, cuestión que, desde una perspectiva mucho más subjetiva, también atribuye la relación de apego entre alguien yalgo.

En tercer lugar, las políticas educativas institucionales, tienen el deber de buscar alternativas para satisfacer las necesidades básicas de mejoramiento psico-afectivo y socio-afectivo del estudiantado. Es decir, si la escuela no adquiere o no asume un verdadero contrato social, que permita encuadrar las relaciones de convivencia del círculo social-humano que le compete, ésta quedaría sumida en un estado político-educativo ficticio, dentro del cual, simplemente se acepta una estructura normativa forzada, que permite la naturalización de la desigualdad, del desconocimiento de la 'particularidad' del ser-humano y del ser-inteligente y de la imposición de dogmas. No se puede pasar por alto, que uno de los principios fundamentales de cualquier proyecto educativo, es ofrecer una educación orientada en favor de la justicia y la participación democrática, mediante procesos funcionales que favorezcan el desarrollo de experiencias de aprendizaje para la vida, coherentes con las necesidades reales de los estudiantes, en favor del reconocimiento del otro y acordes a las demandas de la sociedad actual. (Riádigos, 2014)

De esta manera, frente a los procesos evaluados y a los resultados obtenidos, es posible afirmar que el escaso desarrollo de la competencia socio-afectiva, está afectando el desarrollo intelectual, social y humano de los educandos; lo cual se ve reflejado dentro de un cuadro de estancamiento académico, con alusiones constantes de problemas de convivencia.

\section{Conclusión}

Como bien se ha descrito, la escuela necesita acercarse a un nuevo paradigma educativo, para contrarrestar el estado de estancamiento académico y convivencial, dentro del cual se encuentra. De esta forma, se propone asumir los retos y dinámicas pedagógicas, desde los siguientes cuestionamientos; ¿cómo desarrollar la actitud de querer aprender?, ¿con qué actitud se debe enseñar y cómo se espera que respondan los estudiantes?, ¿qué motivaciones tienen los 
estudiantes para querer aprender? y ¿cómo evaluar su progreso en virtud de su formación como ser-humano? Caso contrario, si la escuela no adquiere o no asume un verdadero contrato social, que permita encuadrar las relaciones de convivencia del círculo social-humano que le compete, ésta podría relegarse a ser un estado político-educativo ficticio, donde simplemente se acepta una normatividad forzada, que permite la naturalización de la desigualdad, del desconocimiento de la 'particularidad' del ser-humano y del ser-inteligente y de la imposición de dogmas. Por lo tanto, la importancia de trabajar un proyecto educativo desde el sujeto como ser-afectivo, se valida en el hecho de permitir a los directivos, docentes, padres de familia y estudiantes, asumir la educación como una experiencia de vida, que permita revolucionar una educación humanista hacía la construcción de una escuela humanitaria.

\section{Agradecimientos}

Un agradecimiento especial a la mesa directiva de FUNDES y en su nombre al Pbro. Ps. Roberto José Guzmán Villanueva (Rector) y al Pbro. Abg. Rodolfo Ramírez Prada (Vicerrector Administrativo). Para la familia Isidorista, al Mg. Gilberto Carvajal Cardoso (Rector), Esp. Jaime Alirio Barrero y Esp. Jesús Eleazar Uribe Páramo, (Coordinadores). Igualmente agradecer a la comunidad educativa de la Institución Manuel Elkin Patarroyo bajo la Rectoría del Lic. Fernando Piza Fernández, así como a la Ps. Mg. Clara Yolanda Gaitán Hurtado. Finalmente, un agradecimiento generoso y sincero, para quienes hacen de la docencia, más que un oficio, una labor de compromiso social y humano. 


\section{Anexos}

\section{Tabla 1}

Análisis reflexivo sobre los procesos de enseñanza aprendizaje

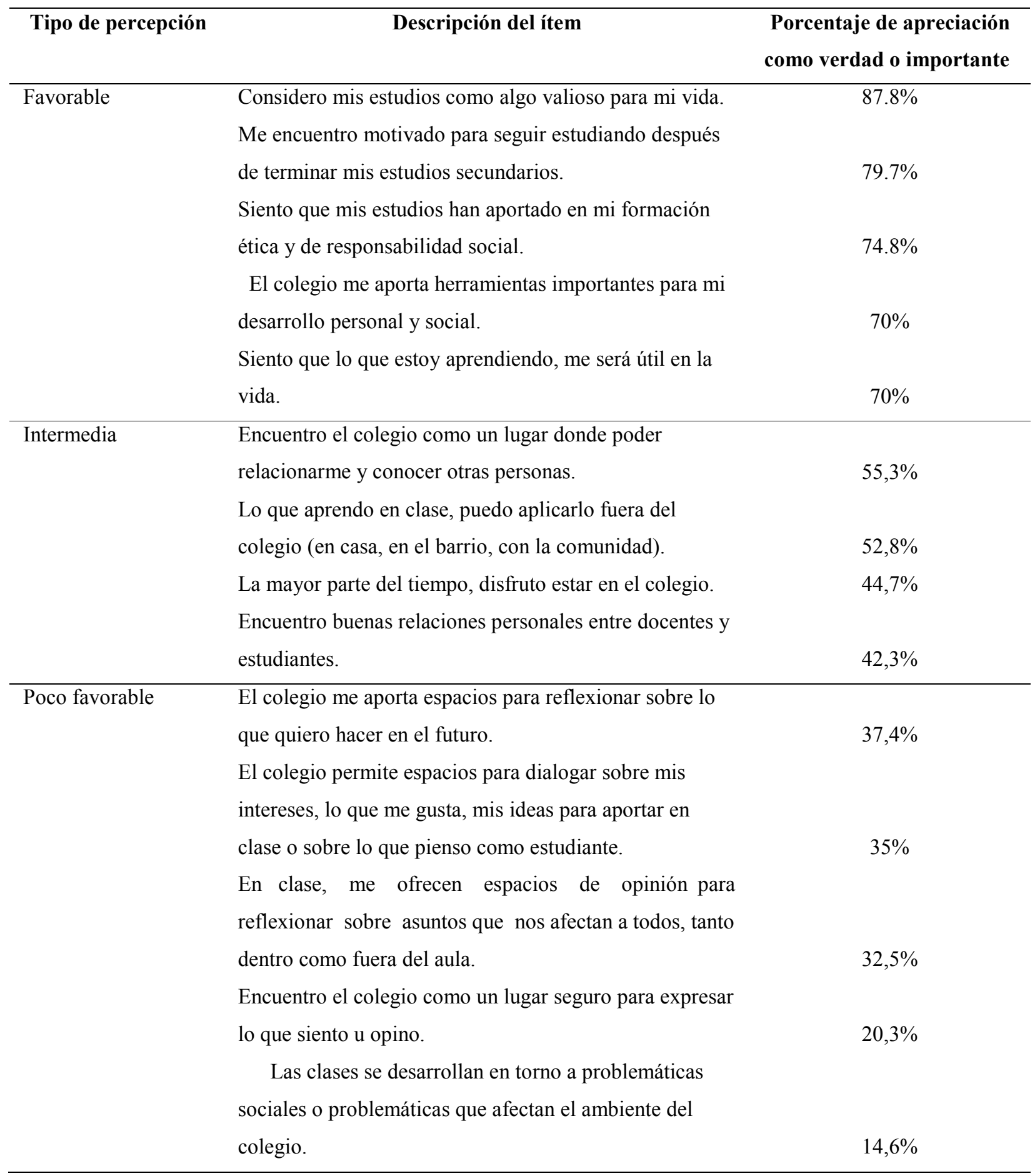

Fuente: Encuesta para identificar factores determinantes sobre los procesos de enseñanza-aprendizaje

(I. E San Isidoro, 2017) 


\section{Referencias Bibliográficas}

Andrade, J.; Torres, D. (2016). Estado del arte del consumo de sustancias psicoactivas en el departamento del Quindío, en el periodo 2009-2012. Drugs and Addictive Behavior, Vol. 1, N¹. (pp. 29-52).

Rescatado

de:

http://www.funlam.edu.co/revistas/index.php/DAB/article/download/1756/1437

Berumen, M.; Arredondo, J.; Ramírez, M. (2016) Revista Ra Ximhai, Vol. 12. N6; juliodiciembre, 2016 (pp. 487-505) Universidad Autónoma Indígena de México. El Fuerte, México.

Rescatado de: http://www.redalyc.org/articulo.oa?id=46148194032

Carpena, A. (2010). Desarrollo de las competencias emocionales en el marco escolar. CEE Participación Educativa $\mathrm{N}^{\circ} 15$. (pp. 40-57)

Rescatado de: http://www2.educacion.es/cesces/revista/n15-carpena-casajuana.pdf

Castañeda, C. (2014). Competencia socio afectiva en el marco escolar colombiano. Revista

Escenarios, Vol. 12. N² (pp.19-34)

Rescatado de: http://ojs.uac.edu.co/index.php/escenarios/article/download/312/287

Diario El Espectador (2013) Tres de cada cinco víctimas de "bullying" en Colombia piensan en suicidio. Nacional. 12 de noviembre de 2013

Rescatado de: https://www.elespectador.com/noticias/nacional/tres-de-cada-cincovictimas-de-bullyingen-colombia-pien-articulo-457937

Diario El País (2014) Acoso escolar afecta a 77,5 \% de los estudiantes colombianos. Noticias.

Cali. Junio 23 de 2014.

Rescatado de:

http://www.elpais.com.co/cali/acoso-escolar-afecta-a-77-5-de-los-estudiantescolombianos.html

Diario El Tiempo (2017) Dos de cada 10 alumnos en el mundo sufren acoso y violencia escolar. Educación. Enero 16 de 2017.

Rescatado de: http://www.eltiempo.com/vida/educacion/acoso-y-violencia-escolar-en-elmundo-37516 
García, B. (2009) Las dimensiones afectivas de la docencia. Revista Digital Universitaria. Vol. 10, $\mathrm{N}^{\circ} 11$. Noviembre 1 de 2009. ISSN: 1067-6079

Rescatado de: http://www.revista.unam.mx/vol.10/num11/art71/art71.pdf

Gobierno Nacional de Colombia (2014) Plan Nacional de Desarrollo para Colombia (PND) 2014 -2018; Capítulo IV: Colombia la más Educada.

Rescatado de: https://www.minagricultura.gov.co/planeacion-controlgestion/Gestin/Plan\%20de\%20Acci\%C3\%B3n/PLAN\%20NACIONAL\%20DE\%20DES ARROLLO\%202014\%20\%202018\%20TODOS\%20POR\%20UN\%20NUEVO\%20PAIS.pdf

Gobierno de Nacional de Colombia (2016) Plan Nacional Decenal de Educación (PNED) 2016 2026.

Rescatado de: http://www.plandecenal.edu.co/cms/images/PLAN\%20NACIONAL\%20DECENAL\%20 DE\%20EDUCACION\%202DA\%20EDICION_271117.pdf

Goleman, D. (2011). Leadership: the power of emotional intelligence, Estados Unidos de América, ama.

Goleman, D. (1998). La práctica de la inteligencia emocional. Traducción del inglés de Fernando Mora y David González Raga. Edit. Kairós. S.A. Numancia 117-121. Barcelona, España. Primera edición. I.S.B.N.:84-7245-407-X. Depósito legal: B-1.843/1999.

Goleman, D. (1995). La Inteligencia Emocional. Buenos Aires: Editorial Javier Vergara. Instituto Nacional de Medicina Legal y Ciencias Forenses de Colombia (2016) Forensis Rescatado de:

http://www.medicinalegal.gov.co/documents/20143/49526/Forensis+2016.+Datos+para+1 a+vida.pdf

Kohlberg, L. (1989) Teoría y práctica del desarrollo moral en la escuela. Rev. Interuniv. Prof. $\mathrm{N}^{\circ} 4$ (pp.79-90)

Rescatado de: https://dialnet.unirioja.es/descarga/articulo/117615.pdf

Márquez, M.; Gaeta; M. (2017) Desarrollo de competencias emocionales en preadolescentes: el papel de padres y docentes. Revista Electrónica Interuniversitaria de Formación del Profesorado. Vol. 20, N² (pp. 221-235) E-ISSN: 1575-0965

Rescatado de: http://www.redalyc.org/pdf/2170/217050478015.pdf 
Martínez, M. (2009) Dimensiones Básicas de un Desarrollo Humano Integral. Polis, Revista de la Universidad Bolivariana, Vol. 8, No 23, 2009 (pp.119-138)

Rescatado de: https://scielo.conicyt.cl/scielo.php?script=sci_arttext\&pid=S071865682009000200006

Montes, M. (2015) Las competencias socio - afectivas docentes y la formación para la práctica educativa del desarrollo personal y para la convivencia, en el marco de la educación inclusiva. Revista nacional e internacional de educación inclusiva ISSN (impreso): 18894208. Volumen 8, Número 3, noviembre 2015

Rescatado de: http://revistaeducacioninclusiva.es/index.php/REI/article/viewFile/101/98

Orcasita, L.; Lara, V.; Suarez, A.; Palma, D. (2018) Factores Psicosociales Asociados A Los

Patrones De Consumo De Alcohol En Adolescentes Escolarizados. Grupo de investigación Bienestar, Trabajo, Cultura y Sociedad. Pontificia Universidad Javeriana Seccional Cali

Rescatado

de:

http://rcientificas.uninorte.edu.co/index.php/psicologia/article/viewFile/7953/11152

Revista Semana (2018) La epidemia de la violencia intrafamiliar: Van cerca de 50.000 casos en 2016. Nación. Familia. Viernes 29 de junio de 2018.

Rescatado de: https://www.semana.com/nacion/articulo/violencia-intrafamiliar-encolombia-2016/492198

Riádigos, C. (2014) El contrato social de la pax capitalis: la necesidad de un juicio educativo en red. Revista Brasileña de Estudios Pedagógicos. Vol. 95, №241, septiembre- diciembre 2014.

Rescatado de: http://www.scielo.br/scielo.php?script=sci_arttext\&pid=S2176$66812014000300002 \& \operatorname{lng}=\mathrm{es} \& \ln \mathrm{ln}=\mathrm{es}$

Santacruz, J. (2015) Correlación entre el consumo de Sustancias Psicoactivas en adolescentes y los factores protectores que lo disminuyen. Universidad Mariana - Boletín Informativo CEI 3 (p.2)

Rescatado de:

http://www.umariana.edu.co/ojs-

editorial/index.php/BoletinInformativoCEI/article/view/1069 
Secretaria de Educación Pública del Estado de México (2014) Manual para el desarrollo de habilidades socioemocionales en planteles de educación media superior.

Rescatado de: http://prepajocotepec.sems.udg.mx/sites/default/files/yna_manual_11.pdf

Segura, M.; Gantiva, A.; Cárdenas, I.; Aguirre, M. (2009) Manual de Desarrollo de

Competencias Afectivas. KIDSAVE-NOUS 2009. ISBN: 978-958-98124-5-7

Rescatado de: http://www.kidsave.org.co/apc-aa-

files/fe5a1a72340dfbec7ec1e477a37f9d1a/Manual_DesarrolloCompAfectivas_4.pdf

Zubiría, J. (2014) Los Modelos Pedagógicos; Hacia una pedagogía dialogante. IV edición.

Editorial Magisterio. ISBN: 978-958-20-1144-4 (p.16) 